\title{
GvHD Lung Obstructive Function Score
}

National Cancer Institute

\section{Source}

National Cancer Institute. GVHD Lung Obstructive Function Score. NCI Thesaurus. Code C131054.

A score for graft versus host disease based on FEV1. 